\title{
Inverse Modeling of Radiative Transfer by Two-Stream Approximation using the Luus-Jaakola Method
}

\author{
J. M. KRIEGER ${ }^{1}$, G. B. LYRA ${ }^{2}$, F. F. FERREIRA ${ }^{3 *}$, W. R. TELLES ${ }^{3,4}$, \\ J. L. SOUZA ${ }^{5}$ and A. J. SILVA NETO 6
}

Received on January 15, 2020 / Accepted on January 28, 2021

\begin{abstract}
The simulation of terrestrial ecosystem processes, using numerical biosphere-atmosphere models that can be coupled to the Atmospheric Models, assist in a better diagnosis and forecast of climate and weather. To be able to represent a particular region, biome or ecosystem, the model parameters need to be fitted for local conditions. This work aims to assess the Luus-Jaakola (LJ) method in the optimization of the parameters in a two-stream radiative transfer model applied to a vegetation canopy. Solar radiation components (incident, $S^{\downarrow}$, and reflected, $S^{\uparrow}$ ) were measured above a sugarcane crop in a Tropical region from February 17 to 24, 2006. Among the combinations of internal and external iterations evaluated for Luss-Jaakola method, 60/30 (external/internal) iterations presented more precise albedo $\left(\alpha=S^{\uparrow} / S^{\downarrow}\right)$ simulated $\left(r^{2}=0.7386\right)$ and, for the accuracy of the simulated $\alpha$, even though the $60 / 40$ combination had the smallest percentual error $(6.40 \%)$, the $60 / 30$ combination was $0.03 \%$ higher. The precision and accuracy of $S^{\uparrow}$ was greater with the parameters obtained by the inverse problem with the combination of $60 / 30$ (external/internal) iterations respectively. In general, the behavior of simulated $S^{\uparrow}$ at the top of the canopy was underestimated compared to the observed $S^{\uparrow}$, especially in the early morning. For the simulated $\alpha$ at the top of the canopy, the model's overestimation was observed at the lowest values of albedo. When the largest albedos are observed, only at the beginning of the day the model underestimated the values. As shown by the tests result, the parameters optimized by Luus-Jaakola method have an adequate representation of the observed data.
\end{abstract}

Keywords: inverse problem, Luus-Jaakola, sensitivity analysis, two-stream model, solar radiation.

*Corresponding author: Fabio Freitas Ferreira - E-mail: fabiofreitasferreira@id.uff.br

${ }^{1}$ Graduate Program in Practices in Sustainable Development, Universidade Federal Rural do Rio de Janeiro, Seropédica, RJ, Brazil - E-mail: joanakrieger@ gmail.com https://orcid.org/0000-0001-8382-2891

2 Departament of Environmental Sciences, Forest Institute, UFRRJ, Seropédica, RJ, Brazil - E-mail: gblyra@ufrrj.br https://orcid.org/0000-0002-9882-7000

${ }^{3}$ Department of Natural Sciences, IHS, Universidade Federal Fluminense, Rio das Ostras, RJ, Brazil - E-mail: fabiofreitasferreira@id.uff.br https://orcid.org/0000-0001-5878-7078

${ }^{4}$ Department of Exact, Biological and Earth Sciences, UFF, Santo Antônio de Pádua, RJ, Brazil - E-mail: wtelles@id.uff.br https://orcid.org/0000-0002-6032-3405

5 Agricultural Science Center, Universidade Federal de Alagoas, Rio Largo, AL, Brazil - E-mail: leonaldojs@yahoo.com.br https://orcid.org/0000-0002-5399-856X

${ }^{6}$ Departament of Mechanical Engineering and Energy, Polytechnic Institute, UERJ, Nova Friburgo, RJ, Brazil - E-mail: ajsneto@iprj.uerj.br https://orcid.org/0000-0002-9616-6093 


\section{INTRODUCTION}

The simulation of terrestrial ecosystem processes, using numerical biosphere-atmosphere models that can be directly coupled to the Atmospheric General Circulation Models (AGCM) and Regional Atmospheric Models, assists to a more accurate diagnosis and prognosis of climate and weather. These models describe the interaction processes with the atmosphere and the transient changes, that occur in vegetation composition, and structure.

Therefore, the constant and current need to improve the understanding and representation of these processes is identified due to the fact that the Brazilian territory, as a whole, has presented different transformations in the spatial pattern of land use and land cover in the last decades [6]. Specially, the solar radiation module of the biosphere-atmosphere models is relevant when considering dynamic models because it is the main mechanism of energy contribution to an ecosystem.

To be able to represent a particular region, biome, or ecosystem, the model parameters are fitted for specific local conditions. These fits receive the name of calibration, which is a set of simulations aiming to optimize the values of the model coefficients so that, if physically consistent, the model describes what is observed. Calibration allows to study each case in detail, so that the models are improved according to the identified needs and available resources [12].

There are several ways of approaching the environmental system in order to obtain the short wave balance, canopy models that link the terrestrial biosphere to the atmosphere can be categorized as multi-layer or big-leaf models. Models such as the big-leaf $[4,8,9,15,22,23]$ are simpler and map the properties of the entire surface, vegetation + soil, treating as a unique leaf. In this set of models, the albedo calculation can be simpler as a unique albedo or more complex as variable hourly albedos and also calculated from theoretical or empirical models. Examples of simpler albedo calculations are the medium or median albedo and of a more complex albedo are, as proposed by Yang (2006 apud [1]) for large plains in the American Southwest and the islands of Manus and Nauru in the tropical Pacific region, the albedo that varies according to the zenith angle as well as the one described by Orsini et al. (2000 apud [1]) for extreme conditions in Reeves Neve Glacier, Antarctica that varies with solar elevation [1]. The multi-layer models, as the two-stream proposed by Coakley and Chýlek [16], are more elaborate and discretize the surface in different homogeneous layers, integrating the flows of each one of them to obtain the total flow.

In this work, the two-stream radiative transfer model applied in a plant canopy, $[3,8]$ was considered. The two-stream model is based on two ordinary differential equations (ODE) solved simultaneously, which estimate radiation flux downward (atmosphere to soil) and upward (soil to atmosphere) in a plant canopy. The model considers in its formulation the attenuation and scattering of radiation in the canopy and treats the spectrum (visible and infrared) and the geometry (diffuse and direct) of radiation separately.

The Shooting Method along with the Euler Method, was used to solve the Ordinary Differential Equations (ODE) system in the Scilab mathematical modeling environment. Before applying 
Inverse Problems techniques to obtain the parameters, the sensitivity analysis of the proposed problem solving model was performed for each of the input parameters. This analysis allowed the identification of the parameters that could be estimated [14].

The Inverse Problem formulation adopted consists on minimizing the functional given by the summation of the squared residues between the calculated values and experimental data, in order to determine the unknown parameters that are present in the direct problem model. There are several techniques to do this, [25], but we will use the Luus-Jaakola method, [18, 24], which showed good results in the previous works $[11,19]$.

\section{FIELD EXPERIMENT}

Solar radiation components (incident and reflected) were measured by a net radiometer (CNR1, Kipp and Zonen, The Netherlands) at the $0.5 \mathrm{~m}$ height above a sugarcane canopy. The net radiometer was connected to a datalloger (CR10X, Campbell Scientific Inc., USA), which measured every $10 \mathrm{~s}$ and stored the average at every $10 \mathrm{~min}$. These data were collected from February 17 to 24,2006 [10].

Radiative flux in the top of canopy was considered equally divided in the solar radiation spectrum between visible (50\%) and infrared (50\%), and ultraviolet radiation was disregarded, due to the small fraction at earth surface [15]. Diffuse radiation was estimated by an empirical model based in the relationship between atmospheric transmissivity - $k t$ (ratio between global solar radiation and extraterrestrial radiation) and the diffuse radiation fraction - $k d$ (ratio between diffuse radiation and global solar radiation). The empirical model was previously fitted by Krieger [17], with experimental data of diffuse and solar radiation from the study area measured by Souza et al. [26].

\section{MATHEMATICAL MODEL}

In this section will be presented the equations that model the solar radiation phenomena. This model is used to prescribe upward and downward radiation fluxes.

\subsection{Upward and Downward diffuse radiative fluxes: Two-Stream}

The model used in this work is based on the two-stream approximation method applied to the radiative transfer equation to obtain a two-fluxes model in plant canopies. All calculations were solved separately for two wavelengths: visible $(0.4$ to $0.7 \mu \mathrm{m})$ and near infrared $(0.7$ to $4.0 \mu \mathrm{m})$.

Within each layer, the upward and downward radiation fluxes [3, 9, 21, 28], respectively, are described by

$$
\begin{gathered}
-\bar{\mu} \frac{\mathrm{d} S^{\uparrow}}{\mathrm{d} L}+(1-(1-\beta) \omega) S^{\uparrow}-\omega \beta S^{\downarrow}=\omega \beta_{0} \bar{\mu} \frac{G}{\mu} D_{0} e^{-G L / \mu}, \\
\bar{\mu} \frac{\mathrm{d} S^{\downarrow}}{\mathrm{d} L}+(1-(1-\beta) \omega) S^{\downarrow}-\omega \beta S^{\uparrow}=\omega\left(1-\beta_{0}\right) \bar{\mu} \frac{G}{\mu} D_{0} e^{-G L / \mu}
\end{gathered}
$$


where, $S^{\uparrow}$ and $S^{\downarrow}$ are the upward and downward diffuse radiative fluxes per unit of area $\left(\mathrm{W} / \mathrm{m}^{2}\right)$, respectively, $L$ is the leaf area index ( $L A I=$ leaf area/ground area) plus the stem area index $\left(S A I=\right.$ stem area/ground area) increasing in the downward direction $\left(\mathrm{m}^{2} / \mathrm{m}^{2}\right), \bar{\mu}$ is the average inverse diffuse optical depth per unit of $\mathrm{d} L\left(\mathrm{~m}^{2} / \mathrm{m}^{2}\right), \mu$ is the cosine of the incident direct beam radiation (cosine of the zenith angle) and $D_{0}$ is its intensity $\left(W / m^{2}\right), G(\mu)$ is the relative projected area of leaf and stem elements in the direction $\cos ^{-1} \mu, \omega$ is the scattering coefficient, and $\beta$ and $\beta_{0}$ are the up scatter parameters for diffuse and direct beam radiation, respectively [21]. The intensity of the direct radiation beam within the layer is given by $D_{0} e^{-G L / \mu}$.

\subsubsection{Boundary conditions}

The required boundary conditions were, for the top of the canopy, where $L=0$, the value of $S^{\downarrow}(0)=S_{0}^{\downarrow}$, which consist on the downward diffuse solar radiation at the top of the canopy, obtained through an empirical model based on the atmospheric conditions (atmospheric transmissivity), see Figure 1. For the upward radiation, which occurs under the canopy, where $L=L_{b}=$ $L A I+S A I$, the value of $S^{\uparrow}$ was given by the equation $S^{\uparrow}\left(L_{b}\right)=S_{L_{b}}^{\uparrow}=\alpha_{D}(\mu) D_{0} e^{-G L_{b} / \mu}+\alpha_{I} S^{\downarrow}$, in which, $\alpha_{D}(\mu)$ and $\alpha_{I}$ are the albedo of the soil for direct radiation in the direction $\mu$ and diffuse radiation, respectively.

\subsection{Direct Problem Solution}

To solve the ODE system, eq. (3.1), the Euler (advanced difference) scheme of the Finite Differences Method (FDM) was used, whose computational mesh was divided into a finite number of layers within the canopy as outlined in Figure 1.

\section{INVERSE MODELING}

In order to minimize the modelling error for the upward radiative flux $S$, the residue between the calculated and the experimental quantity is given by

$$
\mathbf{R}=S_{c}^{\uparrow}-S_{e}^{\uparrow},
$$

where $\mathbf{R}=\left(R_{1}, \ldots, R_{M}\right)^{T} \in \mathbb{R}^{M}$ represents the residue, $M$ is the amount of experimental data, the subscript $c$ indicates the value of $S^{\uparrow}$ calculated computationally and the subscript $e$ indicates the value of $S$ experimentally obtained in the field, $S\left(\mathrm{~W} / \mathrm{m}^{2}\right)$, at the top of the canopy for each simulation time. This method aimed to get a residue as small as possible. Hence, this is a minimization problem.

Therefore, the functional of the square residuals to be minimized is written as

$$
Q(\mathbf{X})=\frac{1}{2} \sum_{i=1}^{M}\left(S_{c_{i}}^{\uparrow}-S_{e_{i}}^{\uparrow}\right)^{2},
$$




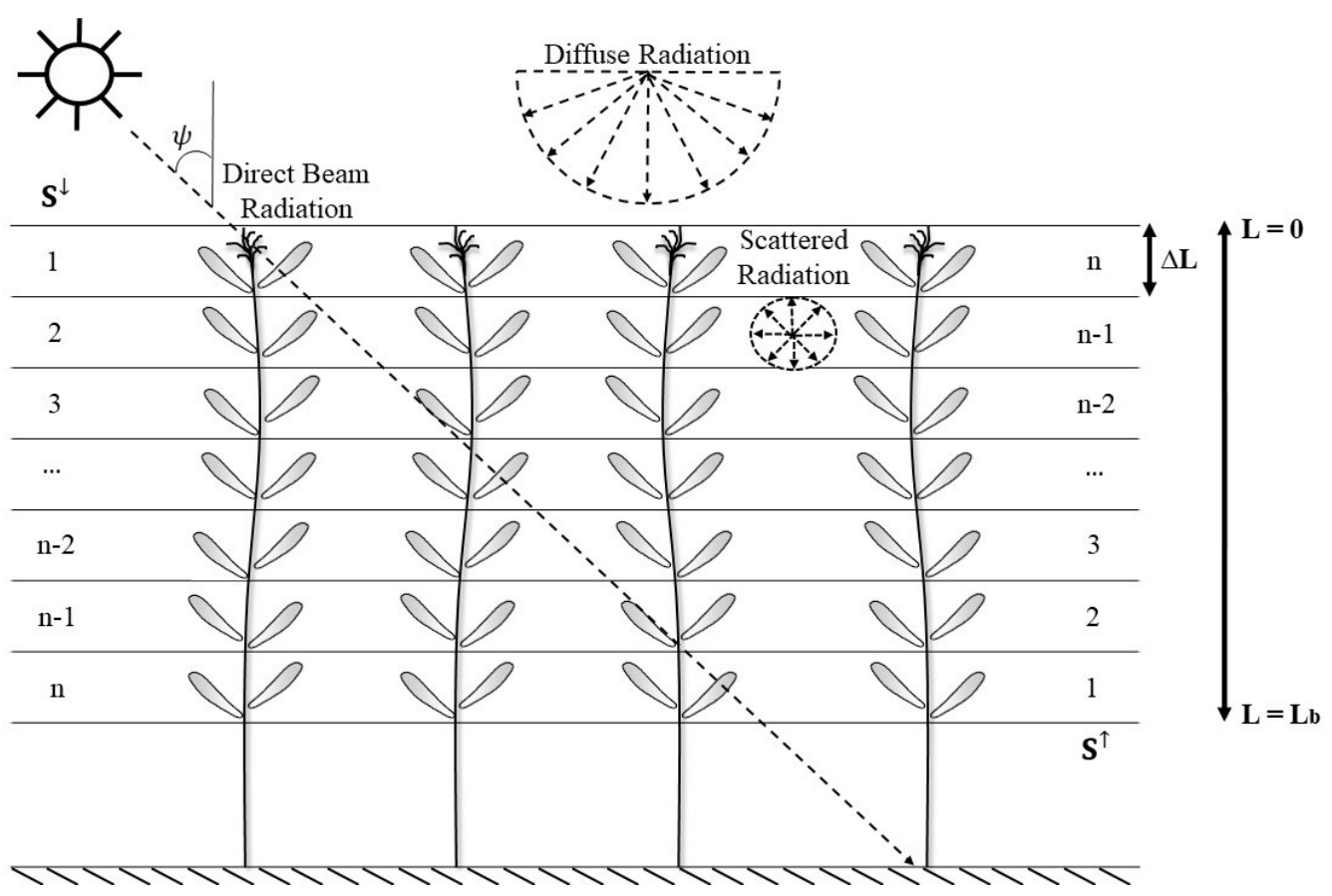

Figure 1: Spatial mesh for the ODE system resolution.

where $\mathbf{X}=\left(\alpha_{I R}, \alpha_{V i s}, \chi, \rho_{I R}, \rho_{V i s}, \tau_{I R}, \tau_{V i s}\right)$ represents the parameters to be estimated [25], $\alpha$ is the albedo, $\rho$ is the reflectivity and $\tau$ is the transmissivity; and the subscript Vis means visible and $I R$ means infrared; and finaly $\chi$ is the leaf orientation.

\subsection{The Luus-Jaakola Method}

R. Luus and T. H. I. Jaakola, [18], developed a simple optimization procedure to solve nonlinear programming problems. The procedure is based on minimizing the functional given by eq. (4.2). For that, the restrictions on the search domain for the parameters were defined according to the existing literature

$$
\operatorname{MIN}_{\mathbf{X}}<\mathbf{X}<M A X_{\mathbf{X}}
$$

This is a minimization problem with restrictions. The Luus-Jaakola method is described in the algorithm below.

1. An initial random estimate is generated, $\mathbf{X}^{0}$, within the restrictions. The amplitude of the search interval $\mathbf{r}^{0}=\operatorname{MAX}\left(\mathbf{X}^{0}\right)-\operatorname{MIN}\left(\mathbf{X}^{0}\right)$ is denoted;

2. The residue $Q_{0}$ according to eq. (4.2) is calculated;

3. The number of times (external iterations) that $\mathbf{r}^{0}$ will be reduced is defined; 
4. A number of times (internal iterations) that random solution candidates will be generated is also defined (parameters to be determined);

5. Random numbers are generated $(\mathbf{Z})$ between -0.5 and +0.5 for each of the parameters;

6. The random numbers from step (5) are taken and assigned to the new $\mathbf{X}$, given by

$$
\mathbf{X}=\mathbf{X}^{*}+\mathbf{Z} \cdot \mathbf{r}, \quad k=1, \ldots, \#(\mathbf{X}) .
$$

where $\mathbf{X}^{*}$ is the best solution of the previous iteration, and $\#(\mathbf{X})$ is the number of parameters to be estimated;

7. The restrictions imposed for $\mathbf{X}$ are tested;

8. The solution of the eq. (3.1) is calculated with the new candidates for the Inverse Problem solution (parameters);

9. The new residue is calculated $Q_{N}(\mathbf{X})$ according to eq. (4.2);

10. If $Q_{N}<Q_{0}$, then $Q_{0}=Q_{N}$. Otherwise, the new values for $\mathbf{X}$ are discarded;

11. The amplitude of the search interval is reduced by a percentage pre-defined in the algorithm, called $\varepsilon$ (for example 0.05), $\mathbf{r}^{i}=(1-\varepsilon) \mathbf{r}^{i-1} \quad 0<\varepsilon<1$;

12. Thus, return to the step (5) until the maximum reduction of $\mathbf{r}^{0}$;

13. At the end of the procedure, the best $\mathbf{X}$ minimizes the functional $Q(\mathbf{X})$.

\section{STATISTICAL ANALYSIS}

The Root Mean Square Error (RMSE), eq. 5.1, was used to assess the $S^{\uparrow}$ estimates after the coefficients optimization. The RMSE represents the average deviation between observed and estimated values. The estimated values obtained with two-stream model were also evaluated using a simple linear regression analysis $\left(Y=\Lambda_{0}+\Lambda_{1} X\right)$, where $X$ is the observed $S^{\uparrow}$ and $Y$ represents the estimated values. Moreover, the determination coefficient $\left(r^{2}\right)$ was calculated using eq. 5.2. Two hypotheses were tested with the Students t test $(p<0.05), H_{0}: \Lambda_{0}=0 ; H_{1}: \Lambda_{0} \neq 0$ and $H_{0}: \Lambda_{1}=0 ; H_{1}: \Lambda_{1} \neq 0$ for $Y=X$.

$$
\begin{aligned}
R M S E & =\left[\frac{\sum_{i=1}^{n}\left(P_{i}-O_{i}\right)^{2}}{n}\right]^{0.5}, \\
r^{2} & =\frac{\sum_{i=1}^{n}\left(P_{i}-O\right)^{2}}{\sum_{i=1}^{n}\left(O_{i}-O\right)^{2}},
\end{aligned}
$$

where $P_{i}$ is the value estimated by the model; $O_{i}$ is the observed value; $O$ is the mean value of the observed values, and $n$ is the number of observations. $r^{2}$ varies from 0 to 1 , where 0 (zero) indicates null precision and 1 (one) represents ideal precision. 


\section{SENSITIVITY ANALYSIS}

In order to analyze the sensitivity of the model to each of the parameters, we used the sensitivity coefficients in eq. 6.1, [7,20]. In [20] is the sensitivity analysis for radioactive transfer problems with integral-differential models.

The scaled sensitivity coefficients are given by

$$
\mathbf{W}_{i}=\mathbf{X}_{i} \cdot \frac{\partial S^{\uparrow}}{\partial \mathbf{X}_{i}} \quad i=1,2, \ldots, N
$$

where $N$ is the total number of problem parameters, and the subscript $i$ indicates the $i$-th parameter. The derivative in the equation (6.1) was calculated using the upward approximation $\left(S^{\uparrow}\left(\mathbf{X}_{i}+\Delta \mathbf{X}_{i}\right)-S^{\uparrow}\left(\mathbf{X}_{i}\right)\right) / \Delta \mathbf{X}_{i}$

The sensitivity coefficient rates are given by

$$
\tilde{\mathbf{W}}_{i j}=\frac{\mathbf{W}_{i}}{\mathbf{W}_{j}}, \quad i \neq j, \quad i=1,2, \ldots, N .
$$

They are instrumental in the evaluation of possible correlations of the parameters to be determined with solution of the inverse problem. When two parameters are correlated, they are more difficult to obtain simultaneously.

\subsection{Sensitivity Results}

This section presents the sensitivity analysis results of parameters. The parameters analyzed are $\alpha_{I R}, \alpha_{V i s}, \chi, \rho_{I R}, \rho_{V i s}, \tau_{I R}$ and $\tau_{V i s}$. Sensitivity analysis allows evaluating if given two distinct parameter sets will have two distinct results. This idea of injectivity allows us to be sure that distinct sets of data in the Luus-Jaakola algorithm will return different results in the presented functional eq. 4.2 .

The parameters $\alpha_{V i s}, \rho_{I R}$ and $\tau_{I R}$ had little sensitivity, see Figure 2. However, the mathematical model is sensitive to all parameters. The correlation between the parameters can be seen in Figure 3.

Input parameters are uncorrelated. So we get all the parameters in the optimization process.

\section{RESULTS AND DISCUSSION}

All the tests of the external and internal iteration combinations in the inverse model, Luss-Jaakola Method, returned values for the parameters with an error less than $10^{-4}$ between observed and simulated data for the period used for the model fit. For each group of parameters obtained by the inverse problem, the tests in the direct model presented results for the reflected solar radiation $\left(S_{\uparrow}\right)$ and albedo $(\alpha)$. Then, this was assessed for its precision, expressed by $r^{2}$, and its accuracy, represented here by the percentage RMSE (Figure 4).

Among the combinations of internal and external iterations tested, the one which resulted in simulated values of more precise albedo $\left(r^{2}=0.7386\right)$ was 60 and 30 external and internal iterations 

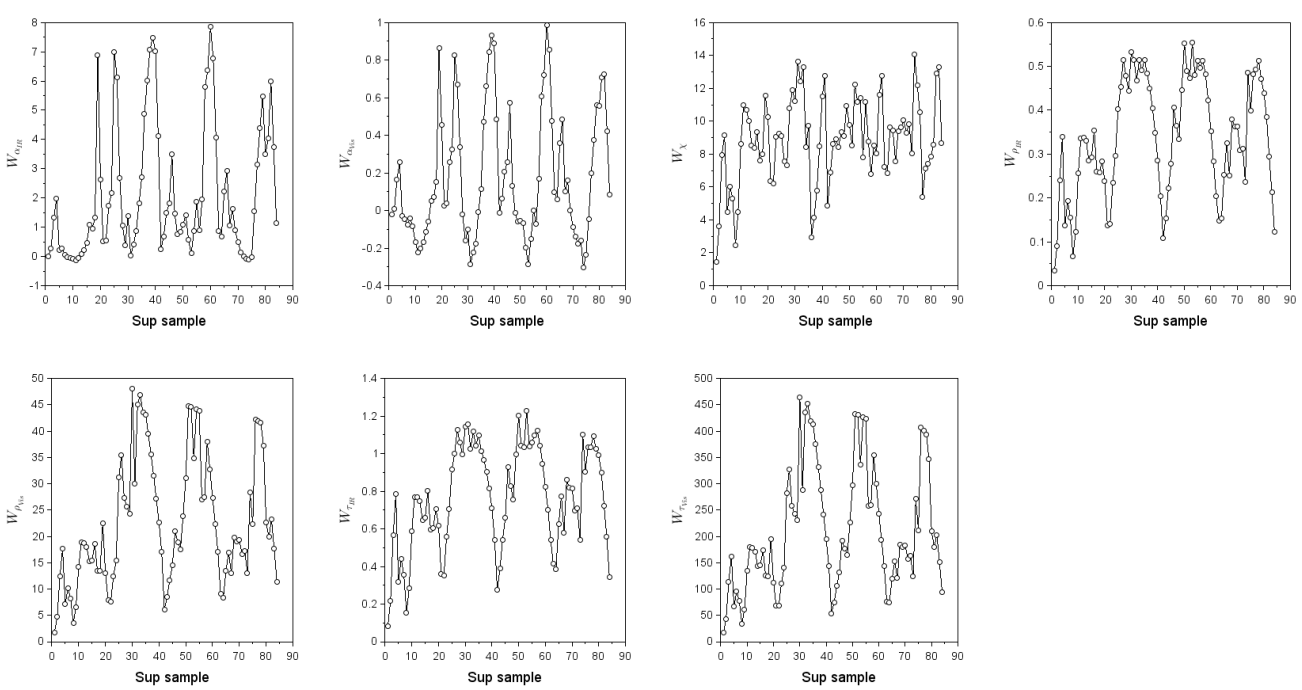

Figure 2: Sensitivity coefficients $\mathbf{W}_{i}$.

respectively. However, as for the accuracy of the combination, 60/40 presented smaller RMSE (Figure 4). Yet, the difference for the 60/30 combination was in the second decimal place. While the $60 / 40$ combination had RMSE equal to $6.40 \%$, the $60 / 30$ combination had $6.43 \%$.

The $r^{2}$ and $R M S E$ of $S^{\uparrow}$ were, respectively, higher and lower with the parameters obtained by the inverse problem with the combination of 60 and 30 external and internal iterations, respectively (Figure 4). The $r^{2}$ obtained by this combination was 0.9934 while the others were below 0.9928 . The error, expressed by percentage $R M S E$ was small for all combinations, but the lowest was obtained with the $60 / 30$ combination, it was equal to $3.54 \%$. Also, comparing the results of the parameters obtained with the ones found in the literature, the present values are physically coherent.

The models using the parameters obtained with the inverse problem solution were tested in the model in the days not used for the optimization (coefficient fits) to get the results for reflected solar radiation and albedo.

The reflected solar radiation was assessed in relation to the established statistical indexes. Although the tests result have an adequate representation of the observed data, in general, the $S^{\uparrow}$ estimated at the top of the canopy for this period (Figure 5) tended to underestimate the values of reflected solar radiation, especially in the early morning $(<7 \mathrm{am})$. It is worth noting that Cuadra [5], worked with a sugarcane crop with the period from $8 \mathrm{am}$ to $5 \mathrm{pm}$, whereas this study used the period from $6: 30 \mathrm{am}$ to $5 \mathrm{pm}$.

The overall performance of the model for the reflected solar radiation in relation to the observed data is also evaluated in Figure $7 \mathrm{c}$ and $7 \mathrm{~d}$. On average, based on the slope coefficient of linear 

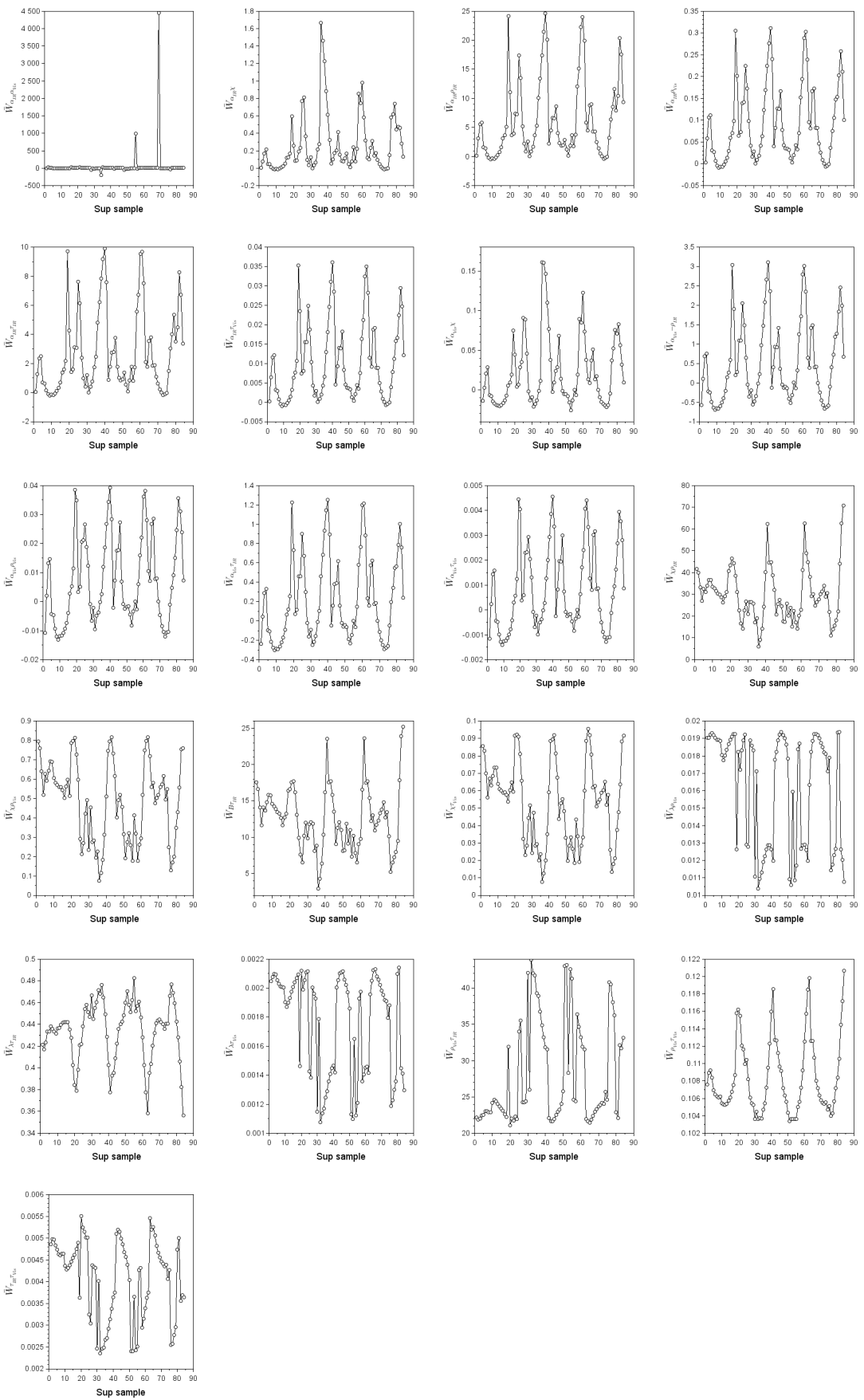

Figure 3: Sensitivity coefficients rates $\overline{\mathbf{W}}_{i j}$. 

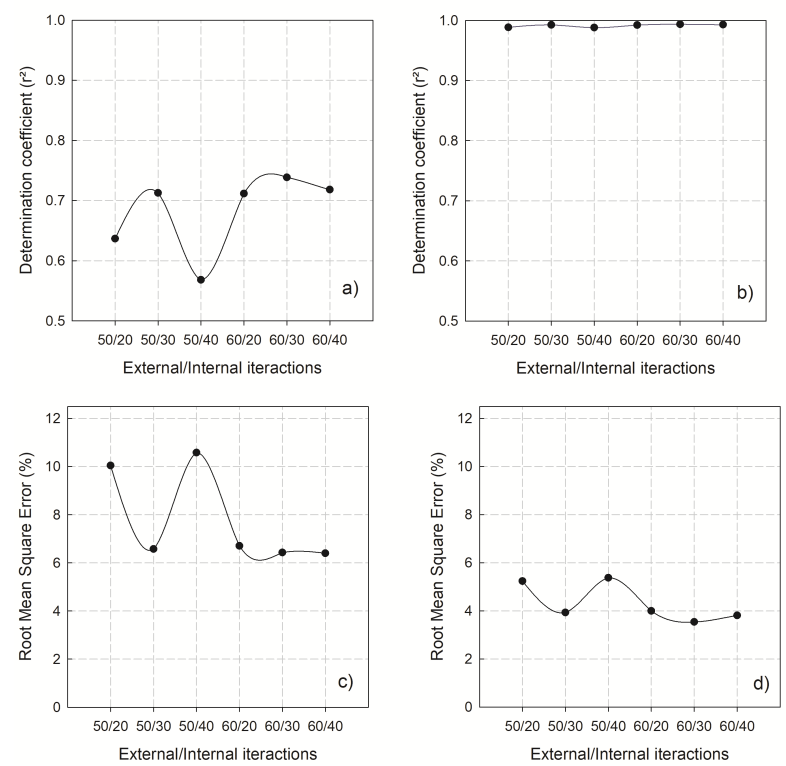

Figure 4: Precision $\left(r^{2}\right)$ (a) albedo and (b) $S^{\uparrow}$; and Accuracy (percentage RMSE) (c) albedo and (d) $S^{\uparrow}$ of the test in the direct model with the parameters obtained considering different configurations for the Luus-Jaakola method.
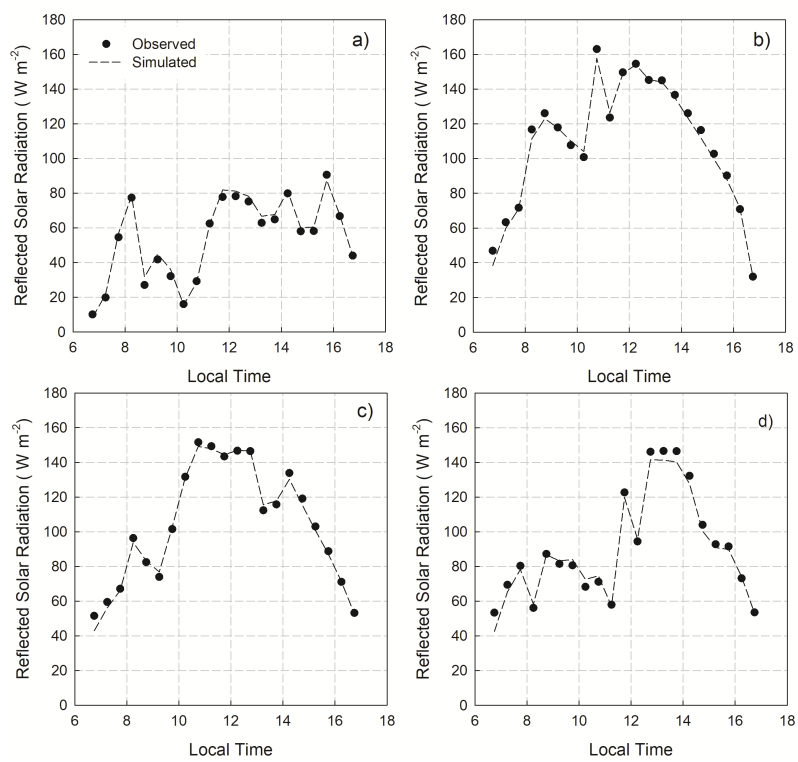

Figure 5: Evaluation of the model performance for the $S^{\uparrow}$ on the top of the canopy for each testing day: a) 02/17/2006, b) 02/18/2006, c) 02/19/2006 and d) 02/20/2006. 
regressions, the model resulted in the underestimation of solar radiation reflected by less than $1 \%$.

Despite being evaluated a few days, Figure 6 showed that the largest errors occurred before $7 \mathrm{am}$. This hour the sun is still low, with cosine of the zenith angle around 0.3 , that is, with zenith angle below $60^{\circ}$. In this case, there is a smaller amount of solar radiation arriving on the surface, due to the longer optical path and greater chances of radiation scattering. So, although it had bigger errors, there were being treated at these times smaller amounts of energy. Before $8 \mathrm{am}$, about $8.5 \%$ of total energy was observed for the days tested. For this reason, many works begin their evaluations from $8 \mathrm{am}$, as in the case of Cuadra [5].

Also, comparing the results obtained using the parameters optimized with the ones found in the literature, the present values are physically coherent. André et al. [2] developed his study with data from an experiment in sugarcane crop installed in the city of Campos dos Goytacazes, Rio de Janeiro, Brazil. In this case, the crop reached the Leaf Area Index (LAI) between 2 and 3 on November 22, 2005 and showed that the peak of reflected solar radiation occurred between 10 and 10:30 $\mathrm{am}$, local time, reaching $202.5 \mathrm{Wm}^{-2}$.

The albedo calculated by $S^{\uparrow}$ estimated at the top of the canopy was also evaluated. In Figure 6 , the model's overestimation was observed at the lowest values of albedo on 17th and 20th of February 2006, for the days beginning at 6:30 am. At the beginning of the day, when the largest albedos are observed, the model underestimated. However, at these times there is less energy available. Yet, at the end of the day, when higher albedos are also observed, the model did not have problems in estimating the values.

Regarding the albedo, Figure 7a shows that the model underestimated for higher albedo values ( $>0.16$ ) mainly early in the day. Also, the model showed a tendency to overestimate albedo values lower than 0.16 . On average, based on the slope coefficient of linear regressions, the model resulted in an underestimation of albedo of approximately $1.5 \%$. When the analysis was made from $8 \mathrm{am}$ (Figure 7b), it was observed that the largest errors that occurred when having high albedo values were no longer observed.

Figure 8, which presents the residue between the observed and estimated data, corroborates the analyzes made. These showed that the biggest errors occurred early in the day, especially before 7 $a m$. It was also confirmed that the largest errors occurred for zenith angle cosine of approximately 0.3 , as previously seen.

Cuadra [5] presented the measured and simulated albedo values for his experiment in sugarcane crop in São Paulo. At the beginning of the day, around $8 \mathrm{am}$, the measured albedo was approximately 0.2 , as well as those simulated for $\chi$ equal to -0.2 and -0.5 . Around noon, the author found the approximate value for the albedo of 0.16 , when he used $\chi$ equal to -0.2 , close to the value of the observed data. However, he obtained albedo values close to 0.14 when he used $\chi$ equal to -0.5 . At the end of the day, around $5 \mathrm{pm}$, the simulated values with $\chi$ equal to -0.2 and -0.5 as well as the values observed for the albedo were approximately 0.21 . 

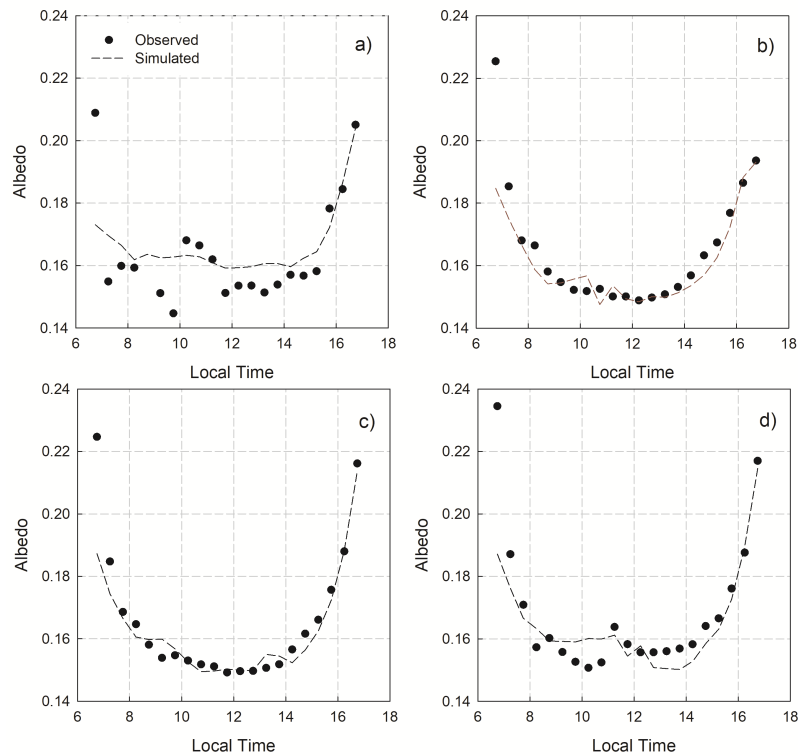

Figure 6: Evaluation of the model performance for the albedo for each testing day: a) 02/17/2006, b) $02 / 18 / 2006$, c) $02 / 19 / 2006$ and d) $02 / 20 / 2006$.
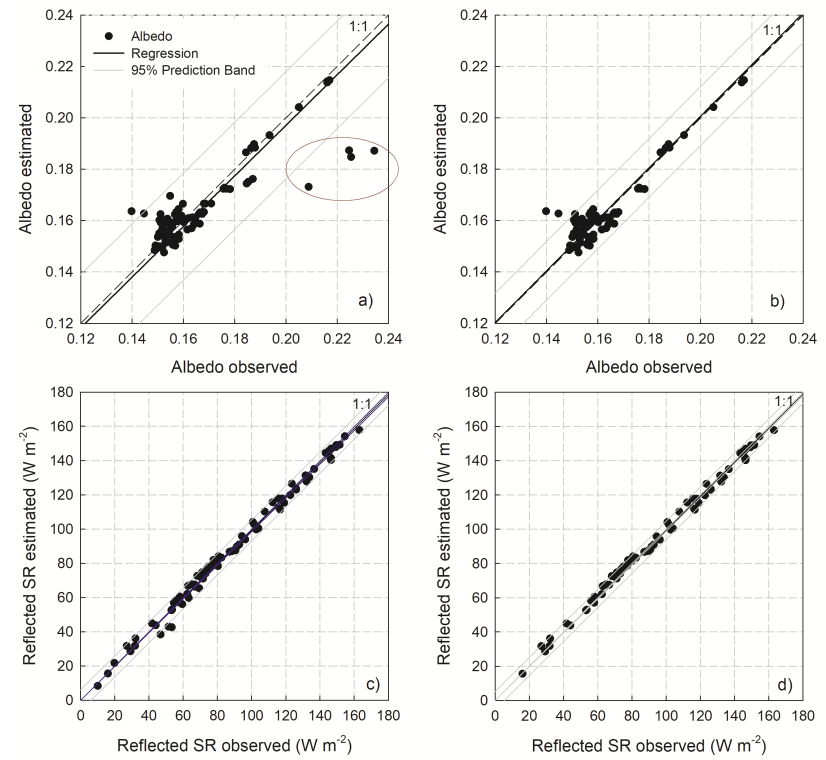

Figure 7: Linear relationship between albedo ( $a$ and b) and reflected solar radiation (c and d) observed and estimated by model starting at 6:30 am (a and c) or starting at $8 \mathrm{am}$ (b and d). 

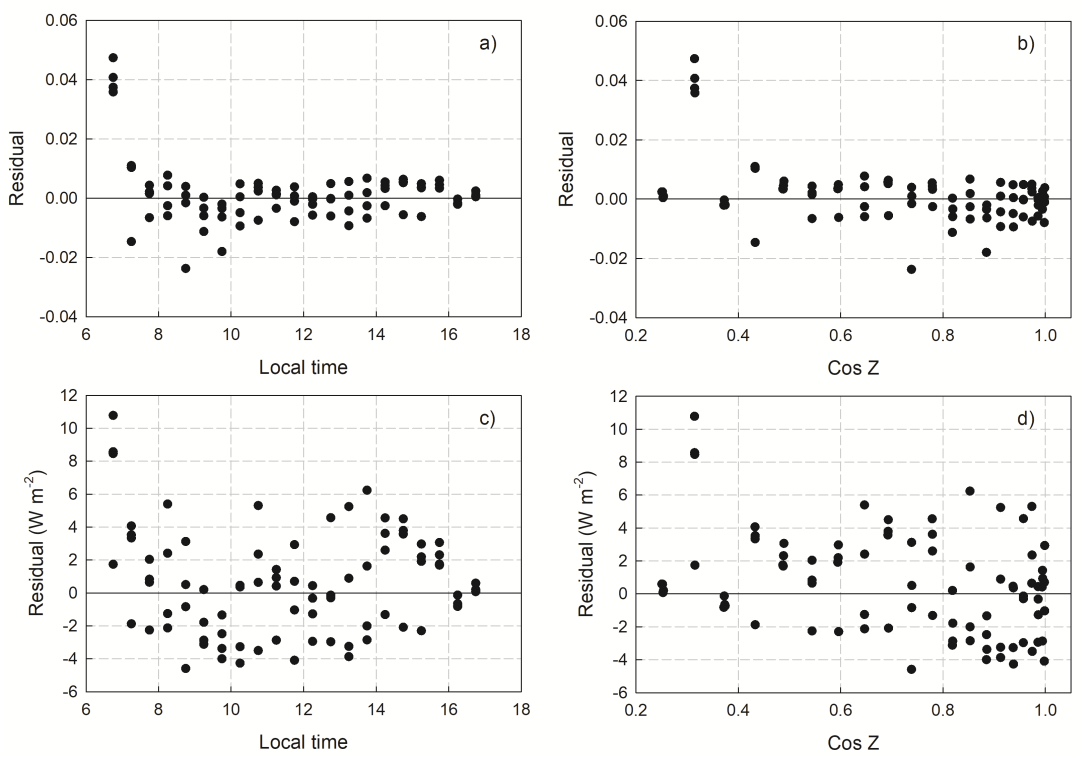

Figure 8: Relation between residue $\left(\mathrm{Wm}^{-2}\right)$, observed minus estimated data, of albedo (a and b) and reflected solar radiation ( $c$ and d) for local time ( $a$ and c) and zenital cosseno (b and d).

André et al. [2] also presented the values found for the albedo on the day observed for the LAI between 2 and 3. In this case, the albedo values varied between 0.23 , around $1 \mathrm{pm}$, about 0.24 in the early morning and 0.28 at dusk. The mean daytime albedo value for this day was $0.23 \pm$ 0.018 .

Gomes et al. [13] obtained albedo values for their experiment also in sugarcane crop in the municipality of Santa Rita do Passa Quatro, São Paulo, Brazil, of 0.18 and 0.20 for February 22 and July 16, 2005, respectively.

Yanagi [27] observed albedo data between 0.12 and 0.16 collected in the Biological Reserve of Cuieiras, brazilian Amazon rainforest, between August and September. The RQME for the albedo estimated by the model using optimized parameters was approximately 0.015 and 0.030 .

\section{CONCLUSIONS}

Among the tests performed with the combinations of internal and external iterations in the LussJaakola model, the result of the most accurate fits obtained for $S^{\uparrow}$ and $\alpha$, when fitting in relation to $S^{\uparrow}$, occurs when using 60 external and 30 internal iterations. When tested for the days not used for the optimization, the parameters obtained by this technique returned values physically coherent, precise and accurate compared with the observed data.

For future studies, the radiative transfer model, as well as numerical methods and the inverse problem method, should be applied to other periods of the year, that is, to use a more repre- 
sentative database to assess the generalization of the model. Although the evaluations for its generalization were not completed, this optimization provided the empirical coefficients.

\section{Acknowledgements}

The authors acknowledge the financial support provided by FAPEAL, FAPERJ, CNPq and CAPES (finance code 001), as well as the graduate programs in Computational Modeling (IPRJ/UERJ) for the opportunity, the Biosystems Engineering (UFF) and the Computational Modeling in Science and Technology (UFF) for the support, and finally, to the Department of Nature Sciences of the UFF for its support to the Postdoctoral Researcher.

\section{Authors contributions}

J.M.K. and G.B.L. conceived the study, coordinated the development, led the analyses and wrote the first version of the study. F.F.F. contributed with the mathematical approach and the sensitivity analyses. W.R.T. contributed with the first version of the Luus-Jaakola script. J.L.S. provided the input data. A.J.S.N. helped analysing the results. All authors provided input into subsequent versions of the manuscript.

\section{REFERENCES}

[1] A. Alfaro, J.M. Krieger, F.L. Albuquerque Neto \& G.B. Lyra. Parametrização do Albedo para Estimativa da Radiação Solar Refletida em Superfície Urbana. In "XX Congresso Brasileiro de Agrometeorologia”. Juazeiro, Bahia e Petrolina, Pernambuco (2017).

[2] R.G.B. André, J.C. MendonçA, V. Marques, F. Pinheiro \& J. Marques. Aspectos energéticos do desenvolvimento da cana-de-açúcar. Parte 1: balanço de radiação e parâmetros derivados. Revista Brasileira de Meteorologia, 25(3) (2010), 375-382.

[3] G.B. Bonan. "Ecological, Hydrological, and Atmospheric Studies: Technical Description and Users Guide" (1996).

[4] G.S. Campbell \& J.M. Norman. "An Introduction to Environmental Biophysics". Springer-Verlag New York, $2^{\text {nd }}$ ed. (1997).

[5] S.V. Cuadra. "Development of a biophysical model of sugarcane growth for global studies (In Portuguese)". Ph.D. thesis, Universidade Federal de Viçosa, Viçosa, MG (2010).

[6] A.P.M.A. Cunha, R.C.S. Alvalá \& G.S. Oliveira. Impacts of vegetation cover changes on surface processes in the semiarid region of Brazil (In Portuguese). Revista Brasileira de Meteorologia, 28(2) (2013), 139-152.

[7] R. de S. Parolin, P.P.G.W. Rodrigues, D.E.M. Domínguez, O.L. Santiago \& A.J. Silva Neto. Sensitivity analysis and estimation of a contaminant source in the Macaé River estuary. Revista Brasileira de Recursos Hídricos, 20(1) (2015), 24-33.

[8] R.E. Dickinson. Land Surface Processes and Climate Surface Albedos and Energy-Balance. National Center for Atmospheric Research, 25 (1983), 305-353. 
[9] R.E. Dickinson, J. Jäger, W.M. Washington \& R. Wolski. "Boundary Subroutine for the NCAR Global Climate Model" (1981).

[10] M.A. dos Santos, J.L. de Souza, G.B. Lyra, I. Teodoro, R.A. Ferreira, A.C. dos Santos Almeida \& M.A.M. Lemes. Observed and modelled solar radiation components in sugarcane crop grown under tropical conditions. International Agrophysics, 31(2) (2017), 231-241.

[11] F.F. Ferreira, W.R. Telles, G.B. Lyra \& A.J. Silva Neto. Comparison of variants of the Luus-Jaakola method in the estimation of van genuchten retention curve parameters. In "Anais do Cobem 2019". Uberlândia, MG (2019).

[12] V.C. Fontes. "Multi-Object and Multi-Object Automatic Calibration of a Terrestrial Surface Model (In Portuguese)". Ph.D. thesis, Universidade Federal de Viçosa, Viçosa, MG (2015).

[13] H. Gomes, B. Silva, E. Cavalcanti \& H. Rocha. Radiation balance in different biomes in the state of São Paulo using Landsat 5 images (In Portuguese). UNESP, Geociências, 28(2) (2009), 153-164.

[14] D.M. Hamby. A review of techniques for parameter sensitivity analysis of environmental models. Environmental Monitoring and Assessment, 32 (1994), 135-154.

[15] M. Iqbal. "An Introduction to Solar Radiation”. Academic Press. The University of British Columbia, Vancouver, Canada (1983).

[16] J.A.C. Jr. \& P. Chýlek. The Two-Stream Approximation in Radiative Transfer: Including the Angle of the Incident Radiation. Journal of the Atmospheric Sciences, (1975).

[17] J.M. Krieger. "Modeling of Radiative Transfer in Sugarcane Crop by Two-Stream Approximation and Finite Difference Method (In Portuguese)". Ph.D. thesis, Universidade Federal Rural do Rio de Janeiro, Seropédica, RJ (2018).

[18] R. Luus \& T.H.I. Jaakola. Optimization by Direct Search and Systematic Reduction of the Size of Search Region. AIChE Journal, 19(4) (1973), 760-766.

[19] R.M.C. Neto, F.F. Ferreira \& A.J. Silva Neto. Assessing van Genuchten's parameters by inverse problems with sensors positioned in different depths and instant (In Portuguese). In "Anais do ENMC 2019”. Juiz de Fora, MG (2019).

[20] R.P.F. Pinheiro, A.J. Silva Neto \& N.C. Roberty. Sensitivity analysis for inverse radiative transfer problem (In Portuguese). In "Proceedings of the 10th Brazilian Congress of Thermal Sciences and Engineering”. Rio de Janeiro, RJ, Brazil (2004).

[21] D. Pollard \& S.L. Thompson. Use of a land-surface transfer scheme (LSX) in a global climate model: the response to doubling stomatal conductance. Global Planet, 10(1) (1995), 129-161.

[22] P. Sellers, Y. Mintz, Y.C. Sud \& A. Dalcher. A simple biosphere model (SiB) for use within General Circulation Models. Journal of the Atmospheric Sciences, 43(6) (1986).

[23] P. Sellers, D. Randall, G. Collatz, J. Berry, C. Field, D. Dazlich, C. Zhang, G. Collelo \& L. Bounoua. A Revised Land Surface Parameterization (SiB2) for Atmospheric GCMs: part I - Model Formulation. Journal of Climate, 9 (1996). 
[24] A.J. Silva Neto, J.C. Becceneri \& H.F. Campos Velho. "Computational Intelligence Applied to Inverse in Radiative Transfer Problems (In Portuguese)”. EdUERJ, Rio de Janeiro (2016).

[25] A.J. Silva Neto \& F.D. Moura Neto. "Inverse Problems: Fundamental Concepts and Applications (In Portuguese)". EdUERJ (2005).

[26] J.L.D. Souza, R.M. Nicácio \& M.A.L. Moura. Global solar radiation measurements in Maceió. Renewable energy, 30(8) (2005), 1203-1220.

[27] S.N.M. Yanagi. "Albedo from an Amazon Rainforest: Field measurements, Remote Sensing, Modeling and its Influence on Regional Climate(In Portuguese)". Ph.D. thesis, Universidade Federal de Viçosa, Viçosa, RJ (2006).

[28] S.N.M. Yanagi \& M.H. Costa. Modeling radiative transfer in tropical rainforest canopies: Sensitivity of simulated albedo to canopy architectural and optical parameters. Anais da Academia Brasileira de Ciências, 83 (2011), 1231-1242. 